\title{
Intra-cerebral implantation of a variety of collagenous scaffolds with nervous embryonic cells
}

\author{
JACEK DROBNIK $^{1}$, KRYSTYNA PIETRUCHA $^{2}$, KAROLINA JANCZAR $^{3}$, LECH POLIS $^{4}$, \\ BARTOSZ POLIS $^{4}$, MARTA SAFANDOWSKA ${ }^{2}$ and JACEK SZYMAŃSKI ${ }^{5}$ \\ ${ }^{1}$ Laboratory of Connective Tissue Metabolism, Department of Pathophysiology, Medical University of Lodz, 90-136 Lodz; \\ ${ }^{2}$ Department of Material and Commodity Sciences and Textile Metrology, Lodz University of Technology, 90-924 Lodz; \\ ${ }^{3}$ Department of Pathomorphology, Medical University of Lodz, 92-213 Lodz; ${ }^{4}$ Department of Neurosurgery, \\ Polish Mothers' Memorial Hospital-Research Institute, 93-338 Lodz; ${ }^{5}$ Central Scientific Laboratory, \\ Medical University of Lodz, 92-215 Lodz, Poland
}

Received October 30, 2018; Accepted August 27, 2019

DOI: $10.3892 /$ etm.2019.8116

\begin{abstract}
Collagenous scaffolds provide good conditions for embryonic nerve cell growth. The aim of the current study was to assess the brains reaction to the implantation of 3D sponge-shaped scaffolds. These scaffolds consisted of collagen $(\mathrm{Col})$ and $\mathrm{Col}$ with chondroitin sulphate, which is modified by carbodiimide, or Col crosslinked with dialdehyde cellulose. The current study also evaluated the expression of integrins $\alpha 2$ and $\beta 1$ in embryonic nerve cells. Embryonic nerve cells were isolated from the brains of rat embryos. Acellular scaffolds, or scaffolds populated with embryonic nerve cells, were implanted into the rats brain. The fibers of all the implanted scaffolds remained intact and served as a template for cell infiltration. The implants induced minimal to moderate inflammatory responses and minimal glial scar formations. Immunohistochemical studies did not indicate any microtubule-associated protein 2 or glial fibrillary acidic protein-positive cells inside the scaffolds. Acellular and cell-populated scaffolds yielded similar responses in the brain. The expression of integrin $\alpha 2$ and $\beta 1$ was observed in embryonic nervous cells. TC-I15, the integrin $\alpha 2 \beta 1$ inhibitor, was not demonstrated to modify cell entrapment within the collagenous scaffolds. All applied scaffolds were well tolerated by the tissue and were indicated to support blood vessel formation. Therefore, all tested biomaterials are recommended for further studies. Additional chemical modifications of the material are suggested to protect the seeded cells from apoptosis after implantation into the brain.
\end{abstract}

Correspondence to: Dr Jacek Drobnik, Laboratory of Connective Tissue Metabolism, Department of Pathophysiology, Medical University of Lodz, Zeligowskiego 7/9, 90-136 Lodz, Poland E-mail: drob@op.pl

Key words: brain injury, scaffold, collagen, chondroitin sulphate, inflammation, integrine, TC-I15

\section{Introduction}

Damage to neurons or glial cells and demyelization after traumatic brain or spinal cord injuries, strokes and neurodegenerative diseases may lead to dysfunction of the nervous system. This phenomenon is linked with serious neurological deficits in patients. The strategies developed for treating injuries or diseases of the central nervous system include pharmacological neuroprotection and tissue engineering. In the latter method, living scaffolds composed of defined cells seeded on biomaterial are implanted to the site of injury (1-3). Several types of scaffolds are used for the treatment of nervous system injuries $(1,3,4)$.

Scaffolds composed of extracellular matrix molecules are thought to be involved in the stimulation of neurogenesis $(1,5)$, a process linked to the functional recovery of a damaged brain. Huang et al (2) report that the implantation of scaffolds containing collagen (Col) and glycosaminoglycans (GAG) provides a good microenvironment for neurogenesis. This data is supported by studies that show scaffolds composed of Col only or of Col with chondroitin sulphate (CS) constitute good environments for the entrapment and cultivation of embryonic nerve cells (6-9). Col scaffolds supplemented with GAG were found to decrease cell adhesion but increase the proliferation of human mesenchymal stem cells (10). Expression of the $\alpha 2 \beta 1$ integrin was confirmed in mesenchymal stem cells seeded within the Col scaffold (11). This integrin is supposed to participate in the adhesion of the cell to Col fibers.

In the present study, three types of Col-based scaffolds were evaluated: Col sponges, bi-component Col-CS matrices crosslinked with 1-ethyl-3-(3-dimethylaminopropyl) carbodiimide hydrochloride (EDC) and Col sponges modified using 2,3 DAC. It has been shown that binary systems of collagenchondroitin sulphate (Col-CS) and collagen-dialdehyde cellulose (Col-DAC) have the correct balance of properties to serve as a biomimetic niche: They accommodate human induced pluripotent stem cells (hiPSC-NPs) sustaining their ability to proliferate and differentiate into neural lineages (12).

The environments of these scaffolds allow the entrapment of nerve embryonic cells and increase their metabolic 
activity $(1,6)$. The aim of the present study was to observe the responses of the brain to different biomaterial implantations (Col, Col-DAC, Col-CS). Both acellular and populated embryonic nerve cell scaffolds were tested through implantation. The longevity of such scaffolds, specifically whether or not they were subject to rapid degradation in the brain, was examined. The study focused on the survival of microtubuleassociated protein 2 (MAP2)- and glial fibrillary acidic protein (GFAP)-positive cells within the scaffolds; it also was evaluated the expression of integrins $\beta 1$ and $\alpha 2$ on embryonic nerve cells, and the involvement of the $\alpha 2 \beta 1$ integrin in cell attachment to the scaffold.

\section{Materials and methods}

Insight into $3 D$ collagen-based scaffolds. Col type I was derived from well-purified porcine tendons by pepsin digestion and acetic acid dissolution to prepare a $0.7 \%(\mathrm{w} / \mathrm{v})$ dispersion. Porcine tendons were bought directly from the slaughterhouse which had proper procedures and permission to obtain this material. Tendons were collected by the company Euroimplant S.A., which was a producer of collagen suspension. Tendons were taken from the lower limbs from fully mature pigs weighting approximately $100 \mathrm{~kg}$, and free from the transmission factors of the infection. Tendons were wrapped in polypropylene foil and frozen at $-18 \pm 2^{\circ} \mathrm{C}$ until to the preparation of dispersion. All experiments were conducted in accordance with national and EU ethical regulations regarding animal free from infections. The use of collagen isolated from porcine tendons was approved by the Local Commission of Ethics in Lodz, Poland (Resolution 4/LB 505/2010).

Three-dimensional (3D) sponge-shaped porous scaffolds were obtained from a dispersion containing Col and CS (purchased from Sigma-Aldrich; Merck KGaA) at a ratio of 100:18. For comparison, sponges from pure Col were also formed. The sponge matrices were formed by freezing the Col dispersion or a mixture of Col with CS and then lyophilized. To improve the functional properties of the scaffold, all sponges were modified according to the methods previously described by Pietrucha (13). In brief, the sponges were immersed in $60 \%$ ethanol solution containing $35 \mathrm{mM}$ 1-ethyl-3-(3-dimethyl aminopropyl) carbodiimide hydrochloride (EDC) and $12 \mathrm{mM} \mathrm{N}$-hydroxysuccinimide (NHS) (EDC and NHS supplied from Sigma-Aldrich; Merck KGaA). Following the reaction, these products were thoroughly washed with $\mathrm{Na}_{2} \mathrm{HPO}_{4}, \mathrm{NaCl}$ and deionized water. To prepare spongy $3 \mathrm{D}$ scaffolds, the matrices were frozen at $-45^{\circ} \mathrm{C}$ and lyophilized at $-55^{\circ} \mathrm{C}$.

The synthesis of the Col-DAC scaffold was performed by cross-linking of Col samples using 2,3-dialdehyde cellulose (DAC). A detailed description of the method has been provided previously (14-16). Briefly, 3D porous Col sponges were incubated in a solution of DAC (synthesized in own scope) at $25^{\circ} \mathrm{C}$ for $24 \mathrm{~h}$ ( $\left.\mathrm{pH} 8.0\right)$. The crosslinked products were thoroughly washed with deionized water and then refrigerated at $-40^{\circ} \mathrm{C}$ and lyophilized at $-55^{\circ} \mathrm{C}$. All of the constructed sponges before implantation were sterilized using an electron beam (accelerator ELU6-Linac) with dose of $18 \mathrm{kGy}$.

Comprehensive descriptions of the biochemical, spectroscopic, morphological and structural properties of the modified Col-based scaffolds can be found in our earlier publications (14-16). Some results of the physicochemical characterization of 3D Col-based sponges are summarized in Table I.

Analysis of the quantitative measurements revealed that both types of investigated scaffolds, i.e., Col-CS and Col-DAC, had an average pore size of approximately $32 \mu \mathrm{m}$. However, the thermal stability and degree of crosslinking of Col-DAC sponges were found to be slightly lower than the Col-CS sponges crosslinked with EDC.

Animals and study design. Thirty male Wistar rats weighing $250 \pm 30 \mathrm{~g}$ and five female $(220 \pm 20 \mathrm{~g})$ were housed with free access to autoclaved commercial food (Murigran, Motycz, Poland) as well as tap water ad libitum. The animals were kept in light (L)-dark (D) conditions (L:D=12:12). The light was switched on at 07.00. The experiments were approved by the Local Commission of Ethics in Lodz, Poland (permission number 37/ŁB617/2012). All experiments were performed according to humane guidelines of legal act from January 15th 2015, devoted to protection of animals used for scientific or educational purposes. The rats were divided into three groups:

Group 1: Rats implanted with Col scaffold

Group 2: Rats implanted with Col-DAC material

Group 3: Rats implanted with Col-CS scaffold

Each group consisted of two subgroups of five rats each. The rats in the first subgroup were treated with an acellular scaffold, while the respective living biomaterial scaffold containing embryonic nerve cells was implanted to the rats in the second subgroup.

Female pregnant rats at 17 days of gestation were used for embryo brain isolation.

Scaffold implantation to the rat brain. Prior to the scaffold implantation rat was anesthetised by intraperitoneal injection of pentobarbital at the dose of $50 \mathrm{mg} / \mathrm{kg}^{-1}$. After anesthesia, the rat was installed in the stereotactic frame, shaved and decontaminated with ethanol. A linear 2-3 cm long skin incision was made and a craniotomy of the left frontal bone of the skull was performed. The respective scaffold was implanted into the frontal lobe of the brain. The skin wound was closed with four sutures. Four weeks after implantation the brains were collected for histological analysis.

Isolation and culture of embryonic nerve tissue. Prior to surgery and embryo collection, the rat was anesthetized by exposure to carbon dioxide $(70 \%)$ and oxygen mixture. The rats were anesthetized in chambers $(25 \times 48 \times 23 \mathrm{~cm})$. Gas flow was $5.6 \mathrm{dm}^{3} / \mathrm{min}$. Then, the animals were euthanized by the spinal cord dislocation. Death of euthanized animals was confirmed by absence of respiration, heartbeat and corneal reflex. Embryonic rats were euthanized by decapitation before brain isolation. The embryos at day 17 of gestation were isolated from the uterus of the pregnant female rat in sterile cold PBS $(7,8)$. Once removed from the embryos, the brains were rinsed with PBS, and the meninges and blood vessels were excised. Then, the brains were incubated in a solution consisting of collagenase $(1 \mathrm{mg} / \mathrm{ml})$ and dispase $(2 \mathrm{mg} / \mathrm{ml})$ for five minutes at room temperature. This step aimed to 
Table I. Characteristics of physicochemical properties of collagen-based sponges.

\begin{tabular}{|c|c|c|c|}
\hline Parameters & Col non-crosslinked & $\begin{array}{l}\text { Col-CS crosslinked } \\
\text { by EDC/NHS }\end{array}$ & $\begin{array}{l}\text { Col crosslinked } \\
\text { by DAC }\end{array}$ \\
\hline Temperature denaturation $\left({ }^{\circ} \mathrm{C}\right)$ & 98.0 & 119.0 & 104.0 \\
\hline Degree of crosslinking (\%) & No crosslinking & $53 \pm 2$ & 49.0 \\
\hline Porosity (\%) & 97.00 & 95.01 & 86.49 \\
\hline Average pore diameter $(\mu \mathrm{m})$ & 55.40 & 31.05 & 32.09 \\
\hline
\end{tabular}

Col, collagen; Col-CS, collagen-chondroitin sulphate; EDC, 1-ethyl-3-(3-dimethylaminopropyl) carbodiimide hydrochloride; NHS, N-hydroxysuccinimide; DAC, 2,3-dialdehyde cellulose.

remove the connective tissue. The samples were then incubated with trypsin for five minutes at $37^{\circ} \mathrm{C}$. After trypsin neutralization, the samples were centrifuged at 1,000 rpm. Following this, the cells were suspended with $100 \mathrm{ml}$ of medium (MACS NeuroBrew-21; MiltenyBiotec) and triturated three to five times gently. Cell suspension was seeded on laminin-covered dishes. For cell cultures, the medium composed of MACS NeuroBrew-21, gentamycin $(25 \mu \mathrm{g} / \mathrm{ml})$ and fungizone $(2.5 \mu \mathrm{g} / \mathrm{ml})$ was applied. The cells were packaged on the scaffold at a density of $9 \times 10^{3}$ cells/sample in 96 well plates (7). After three days of cultivation, the scaffolds were implanted into the brains.

The isolated cells were identified in the earlier studies $(7,8)$. The embryonic nerve cells were nestin negative but they were morphologically differentiated $(7,8)$.

Flow cytometry experiments. To confirm the differentiation of the isolated embryonic nerve cells into neurons (MAP 2 positive cells) as well as the expression of integrins, flow cytometry experiments were performed. After fixation (Fixation Buffer, BDCytofix), the cells were permeabilized by BD Phosflow Perm Buffer III. Following this, the cells were washed and centrifuged $(5 \mathrm{~min}, 1,000 \mathrm{rpm})$ and then stained $\left(4^{\circ} \mathrm{C}-8^{\circ} \mathrm{C}\right.$ for $30 \mathrm{~min}$ ) in Stain Buffer (BD Pharmingen). After washing and centrifugation (for $10 \mathrm{~min}$ at 2,000 rpm) the cells were treated with the following antibodies: mouse anti-MAP2 conjugated with Alexa Fluor 488, mouse IgG1 k isotype control conjugated with Alexa Fluor 488 (BD Pharmingen) Hamster IgG211 isotype control conjugated with FITC, Hamster anti-rat CD49b conjugated with FITC (BD Pharmingen), Arm Hamster IgG isotype control conjugated with $\mathrm{PE}$, anti-mouse/rat CD29 conjugated with PE (eBioscience). The cells were then analyzed using a FACScan analytical flow cytometer (Becton Dickinson) and the results were compared with the isotype control.

Investigation of the involvement of integrin $\alpha 2 \beta 1$ in cell entrapment within the collagenous scaffolds. The nerve embryonic cells were applied into the Col scaffolds seeded into the 96-well plates at a density of $9 \times 10^{3}$. The cells were incubated for $30 \mathrm{~min}$ at $37^{\circ} \mathrm{C}$ with either the medium only, the medium containing $0.1 \%$ of DMSO (the TC-I15 solvent) or the medium containing the TC-I15 inhibitor of the $\alpha 2 \beta 1$ integrin $\left(10^{-5} \mathrm{M}\right)$. After $30 \mathrm{~min}$, the samples were triturated 30 times to wash away the unbound cells. After that time, the cells were stained with bisbensimide $(1 \mu \mathrm{g} / \mathrm{ml})$. The cell nuclei were counted under a fluorescent microscope at magnification, $\mathrm{x} 100$. Each investigated group contained eight samples.

Histopathology. Following dissection, the brains were fixed in formalin. For further evaluation, coronal sections of each brain with grossly visible alternations (cavity) were selected. In cases with no macroscopic changes, the whole brain was submitted for standard tissue processing. Sections with a thickness of $3 \mu \mathrm{m}$ were cut from blocks of formalin-fixed paraffin-embedded tissue, mounted on microscopic slides and submitted for standard hematoxylin and eosin staining and immunohistochemistry.

Immunohistochemical staining was performed to identify neurons and dendrites (microtubule associated protein $2 \mathrm{a}, \mathrm{b}$, c, MAP2) and to show the astrocytes (glial fibrillary acidic protein, GFAP). For antigen retrieval and deparaffinization, the slides were heated for $20 \mathrm{~min}$ at $97^{\circ} \mathrm{C}$ in EnVision Flex Target Retrieval Solution (high pH; Dako) in the PT-link instrument (Dako). For the GFAP immunostaining process, the pre-treated slides were loaded into the autostainer instrument (EnVision System; Dako) and stained with the FLEX polyclonal rabbit anti- GFAP ready-to-use primary antibody (IR524; Dako). For the MAP2 immunostaining, following pre-treatment, the slides were incubated with Peroxidase Blocking Reagent (Dako) for five minutes, and then with the primary monoclonal mouse anti-microtubule associated protein 2 (MAP2, MA5-12826, 1:200; Thermo Fisher Scientific, Inc.) for $16 \mathrm{~h}$ at $4^{\circ} \mathrm{C}$. The MAP2 staining was visualised using the Dako Real EnVision Detection System (Dako). After visualisation, the slides were counterstained with hematoxylin, then dehydrated, cleared in xylene and mounted.

\section{Results}

Histological evaluation. Collagen cross-linked with EDC implanted into the brain. Among the sustained collagenous fibers of the scaffold, an inflammatory infiltrate was composed of mononuclear cells (macrophages and lymphocytes), multinucleated giant cells (foreign body type macrophages) and a few polymorphonuclear cells (Fig. 1A). Moreover, clusters of the meningeal-like cells were found within the scaffolds. Blood vessels (Fig. 1B) were seen in some scaffolds implanted into the brain. Neither MAP2-positive (Fig. 1F) nor GFAP-positive cells (Fig. 1E) were found within the scaffold. 
In the adjacent area of the injury, brain mononuclear cells (several lymphocytes, some hemosiderin-laden macrophages and a few macrophages) and reactive astrocytes were observed. Thick cerebral leptomeninges contained several lymphocytes and macrophages, some of which were laden with hemosiderin. In the deeper part of the brain, small basophilic structures (dying neurons) were found (Fig. 1B). In the adjacent area, reactive mononuclear cells (MAP2 negative) were observed, as well as an increased number of GFAP positive (glial) cells (Fig. 1E).

Implantation of collagen cross-linked by dialdehyde cellulose. Numerous cells were found to have infiltrated the implant fibers; the inflammatory cells were thus comprised of lymphocytes, multinucleated giant cells, macrophages and neutrophils (Fig. 1C). Furthermore, calcifications and eosinophilic structures were observed within the scaffolds. Thin-walled blood vessels were shown in some implanted scaffolds.

In the brain surrounding the implant, focal edema and gliosis were observed. Moreover, hemosiderin deposits, amyloid bodies, small calcifications, hemosiderin-laden macrophages and dispersed infiltration of mononuclear cells (lymphocytes) were detected (Fig. 1C). Fibrosis of the cerebral leptomeninges was present. In the adjacent area of the brain, a thin layer of MAP2 negative mononuclear cells was observed (Fig. 1G).

Implantation of composite containing collagen supplemented with CS cross-linked by EDC. Numerous cells were found among the fibers of the implanted scaffold, these being mononuclear cells (macrophages, hemosiderin-laden macrophages and lymphocytes) and multinucleated giant cells (foreign body type' macrophages) (Fig. 1D). Thin-walled blood vessels were seen among the collagen fibres of a few scaffolds implanted into the brain. In the area adjacent to the implant layer, hyperaemia was observed, as were small hemosiderin deposits and mononuclear cells (hemosiderin-laden macrophages, macrophages and lymphocytes). Moreover, cerebral leptomeninges, found adjacent to the cavity containing the implant, showed features of fibrosis. In the deeper parts of the brain area, small, oval, basophilic structures were observed (Fig. 1D). In the adjacent brain area, a thin layer of MAP2 negative mononuclear cells was observed (Fig. 1H).

Examination of the adhesion of cells to the scaffold. Expression of both integrin $\alpha 2$ (Fig. 2A) and integrin $\beta 1$ (Fig. 2B) was detected on nerve embryonic cells by flow cytometry studies. The same numbers of entrapped cells were observed in the samples incubated with TC-I15 in the collagenous scaffold (Fig. 2C), the control group and the cells treated with TC-I15 solvent $(0.1 \%$ DMSO).

\section{Discussion}

Our results confirm the presence of $\mathrm{Col}$ fibers of the inserted scaffold within the area of the brain injury, with the morphological features of the scaffolds appearing to remain intact. The scaffolds remained structurally intact for as long as four weeks after implantation, suggesting that the material may serve as a suitable template for cell migration. Moreover, the fibers of the graft may be involved in the regulation of electrolytes and water homeostasis of the tissue (17).
The implanted material induced no or minimal inflammatory reaction in adjacent brain areas. The inflammatory cells were found to accumulate around the scaffold as well as infiltrated into the biomaterial (Fig. 1A-D). Implantation of the scaffolds induced granulomatous tissue reaction comprising an accumulation of multinucleated macrophages, mononuclear inflammatory cells and formation of blood vessels. These results are comparable with other studies. The inflammatory reaction was seen to be calmed by implantation of gelatin-laminin materials (18), while Vaysse et al did not find any inflammatory reaction three months after implantation of micropatterned polydimethylsiloxane with neuronal cells, with the graft remaining intact (19). No excessive inflammatory reaction was found to be associated with any of the three types of applied implants during the present study (Fig. 1A-D).

Vascularization is a critical and important event in tissue engineering. In all three types of tested biomaterials, thin-walled blood vessels were found to be situated within the scaffolds. These results suggest that $\mathrm{Col}$ alone, independent of the cross-linking method, may support new blood vessel formation. Hence, even without addition of angiogenic factors, the tested samples constituted a suitable niche for promoting local angiogenesis. Scaffolds with angiogenic properties are recommended in the treatment of ischemic brain lesions (20). Some reports suggest a relationship between neurogenesis and angiogenesis in brain lesions (21). Huang et al (5) report increased angiogenesis after Col-GAG scaffold implantation into the brain; however, the types of GAGs forming the scaffolds were not defined. Elsewhere, implantation of such as scaffold into the brain lesion increased proliferation of both smooth muscle cells and endothelial cells (22). These phenomena were accompanied with an increased concentration of angiogenic growth factors (VEGF, FGF2, PDGF) in the tissue $(2,5)$. To fully clarify the angiogenic properties of the scaffolds, further studies should employ immunohistochemical staining of CD 44.

Little development of the glial scar at the graft periphery was observed, but no GFAP positive cells (cells with glial differentiation) were found inside the scaffold (Fig. 1E); in addition, no excessive gliosis was observed after any scaffold implantation. These results are supported by those of experiments performed on other scaffolds $(2,18,23)$. After implantation of the Col-GAGs scaffold into the brain, astrocite accumulation was reported only in the lesion boundary zone, and these cells did not proliferate (2). Jurga et al found that astrocites mainly accumulated at the edges of the scaffolds (18). Although infiltration of astrocite microglial and endothelial cells was reported following the introduction of a gelatin-siloxane biomaterial implant into the brain, no infiltration of neurons was reported (19).

Flow cytometry studies have found that the embryonic nerve cells seeded within the scaffolds contained cells differentiating into neurons (MAP2-positive cells) $(7,8)$; these are able to replace the damaged nervous tissue at the site of trauma, and are hence, crucial for tissue reconstruction. Scaffolds populated with embryonic nerve cells are also believed to be a source of cytokines that may accelerate the regeneration process. However, the clinical effects of cell-containing scaffold implantations into the brain are very limited due to low cell survival rate: the cells that populate the scaffolds usually die a few days after implantation. The main causes 
A

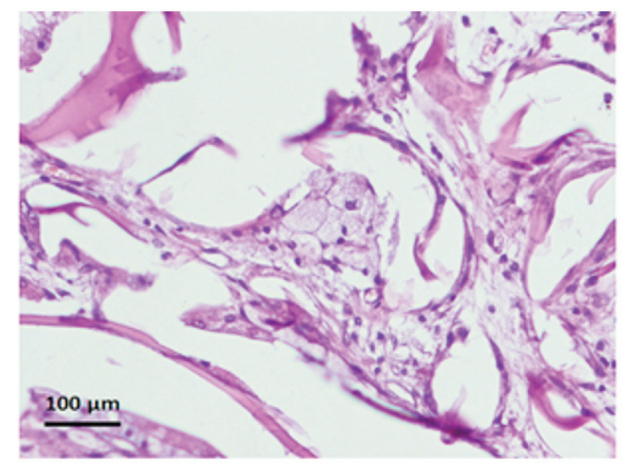

C

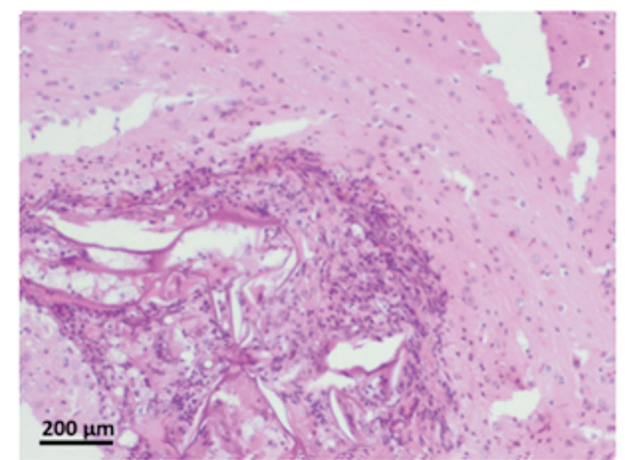

$\mathbf{E}$

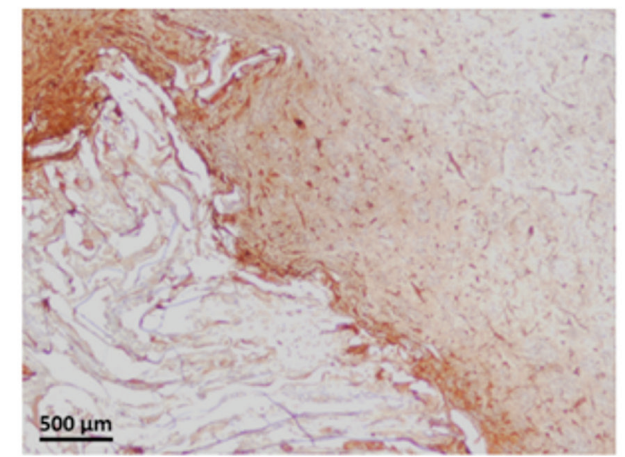

G

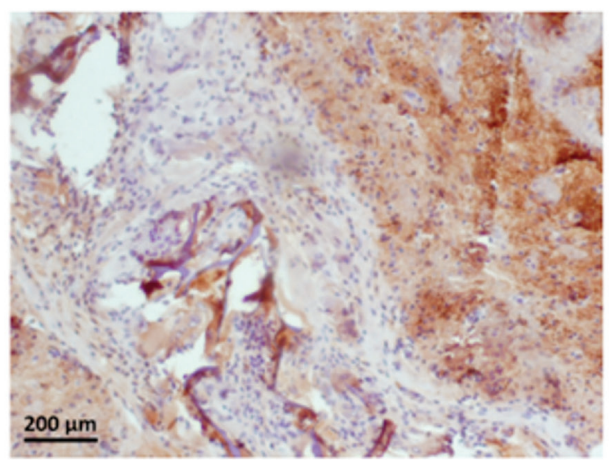

B

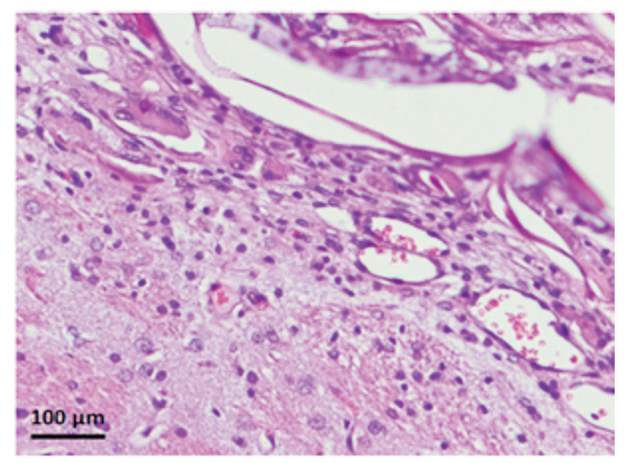

D

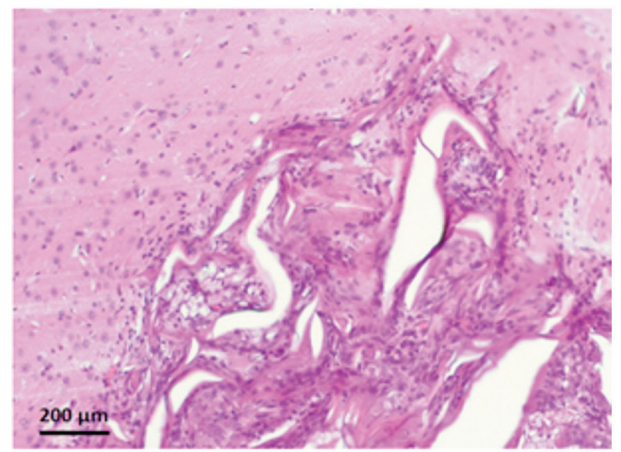

$\mathbf{F}$

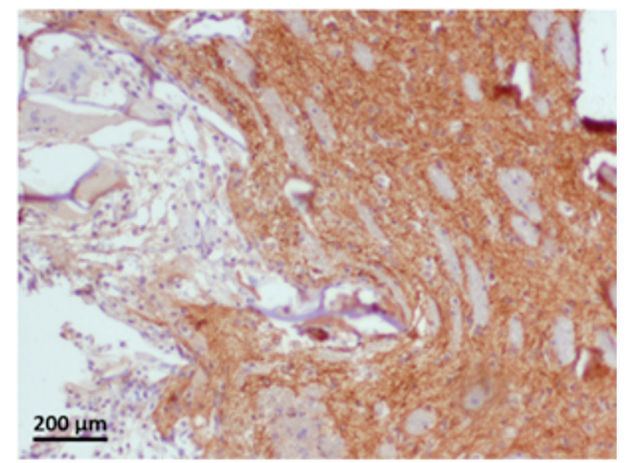

H

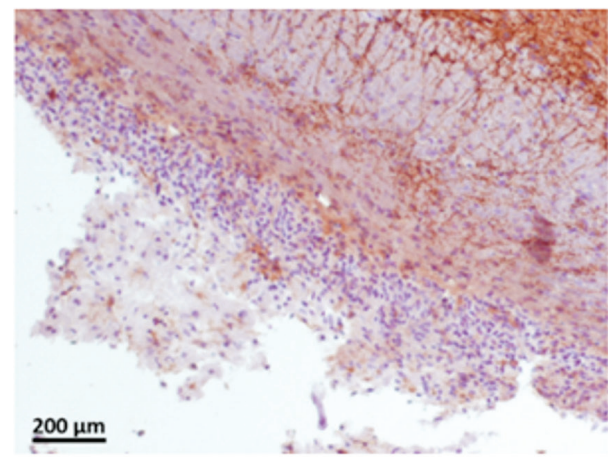

Figure 1. (A) Col scaffolds grafted into the brain. Among the sustained collagen fibers, multinucleated, macrophages (cells with foamy cytoplasm) and mononuclear inflammatory cells (lymphocytes) are observed. Staining with hematoxylin and eosin. Magnification, x200. (B) The brain adjacent to the Col scaffolds. Multinucleated giant cells and thin-walled blood vessels are present among the implanted collagen fibers (right upper part of the figure). Mononuclear inflammatory cells (lymphocytes) in the adjacent brain. Staining with hematoxylin and eosin. Magnification, x200. (C) The brain adjacent to the Col-DAC scaffolds. The implanted Col-DAC scaffold (left lower part of the figure) is infiltrated by multinucleated giant cells and mononuclear cells (lymphocytes and macrophages). The reaction of the adjacent nervous tissue to graft implantation is minimal and comprises mononuclear cells (lymphocytes). Staining with hematoxylin and eosin. Magnification, x100. (D) The brain tissue adjoined with the Col-CS scaffolds. The implanted Col-CS grafts (right lower part of the figure) contain multinucleated giant cells and mononuclear cells (lymphocytes and macrophages). The reaction of the tissue to graft implantation is minimal and comprises mononuclear cell infiltration. Staining with hematoxylin and eosin. Magnification, x100. (E) GFAP immunostaining of the brain adjacent to the Col scaffolds. Cells present among the implanted collagen fibers (left lower part of the figure) are GFAP-negative. The positive GFAP reaction can be observed in the surrounding brain. Magnification, x40. (F) MAP2 immunostaining adjacent to the Col scaffolds brain. The tissue surrounding the Col graft is normal and indicated MAP2 immunoexpression. The mononuclear cells directly adhered with the scaffold remnants and the multinucleated giant cells within the graft are MAP2-negative. Magnification, x100. (G) MAP2 immunostaining of the brain adjacent to the Col-DAC scaffolds. Cells directly adhered the Col-DAC scaffold and those situated within the graft are MAP2 negative. The MAP2-positive reaction is seen within the normal brain. Magnification, x100. (H) MAP2 immunostaining of the brain adjacent to the Col-CS scaffolds. A thin cell layer directly adhered the Col-CS scaffold and those situated within the graft were revealed to be MAP2 negative. The MAP2-positive reaction is observed within the encephalon. The scaffold is not visible in the image. Magnification, x100. Col, collagen; Col-DAC, collagen-dialdehyde cellulose; Col-CS, collagen-chondroitin sulphate; MAP2, microtubule-associated protein 2; GFAP, glial fibrillary acidic protein. 

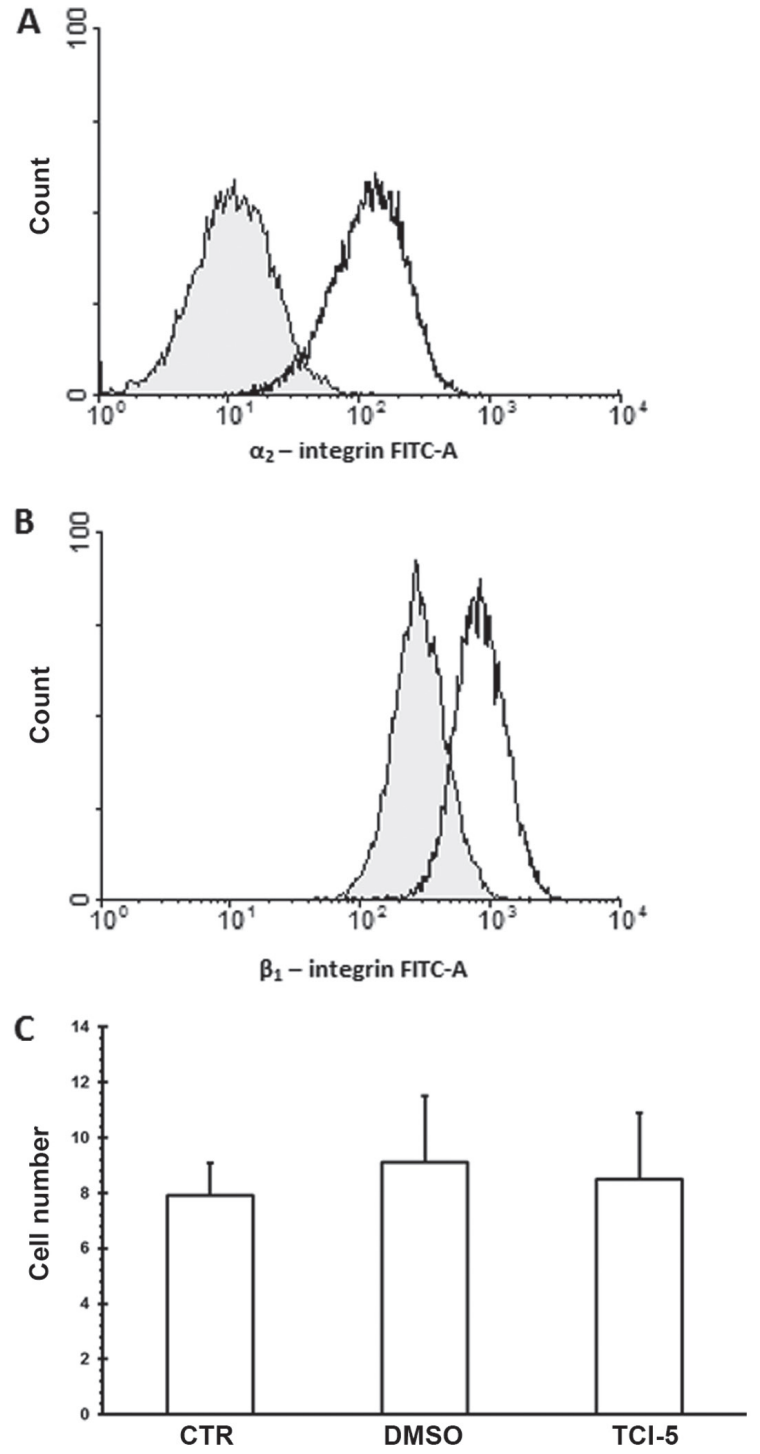

Figure 2. (A) FACS profiles indicating embryonic nervous cell expression of $\alpha 2$ integrin (right curve) compared with isotypic control (left curve). (B) FACS profiles demonstrating the expression of b2 integrin on embryonic nervous cells (right curve) compared with isotypic control (left curve). (C) Cell number seeded on collagenous scaffolds incubated with medium (CTR), $0.1 \%$ DMSO or TCI-15 integrine $\alpha 2 \beta 1$ inhibitor $10^{-5} \mathrm{M}$ (TCI-15). The cells were counted in five microscopic fields of each sample $(\mathrm{P}=\mathrm{x} 100)$. Each value expresses the mean of seven samples \pm standard deviation.

of cell death are inflammation, immune processing, oxidative stress and low levels of trophic factors $(23,24)$. The main mechanisms of neuron death are necrosis and apoptosis (25). Sortwell et al (26) found that the critical period for apoptotic death is the first four days post-implantation. In the present study, no MAP2-positive cells were found within any scaffold at four weeks after implantation (Fig. 1F-H). Furthermore, no additional effects with regard to brain injury regeneration were observed in the cell-seeded scaffold compared with the acellular scaffold. This lack of difference may be linked to fact that these cells populated within the scaffolds are believed to die just after implantation.

Our present observations regarding cell survival reflect a problem described earlier in the literature $(25,26)$. Although all tested scaffolds were well tolerated by injured brain tissue, they were unable to shelter seeded embryonic nerve cells from death. Applied scaffolds cannot support the survival of the embryonic nerve cells or the ingrowth of MAP2- or GFAP-positive cells from adjacent brain. Moreover, observations in vivo revealed that meningeal cells and blood vessels may grow into the scaffolds. Thus, the additional modification of the sponges are postulated to create a niche supporting embryonic nerve cell survival in vivo.

Some earlier studies were performed to protect the cells populated within the scaffold from death. Marchionini et al (27) tested the effects of caspase inhibitors in protecting tyrosine hydroxylase immunoreactive neurons from apoptosis, but found neuron survival not to be prolonged. Despite this, a recent study by Vaysse et al did achieve neuron survival following the implantation of micropatterned polydimethylsiloxane biomaterial with neuronal cells into the brain. This improvement in the neuronal cell survival resulted in the addition of polylysine and laminin to the graft (19). These compounds are believed to reduce the traumatism of implantation. All grafts used in our study displayed beneficial effects for embryonic nerve cell growth, and have previously been reported in in vitro experiments $(7,8)$. However, the tested samples were unable to improve the survival of the cells within the grafts after implantation into the brain.

The interaction of the Col with embryonic nerve cells may influence both the differentiation and the migration of the cells $(18,24,28,29)$. Our findings are the first to demonstrate the expression of both $\alpha 2$ and $\beta 1$ integrins on the surface of the embryonic nerve cells (Fig. $2 \mathrm{~A}$ and B). Integrin $\alpha 2 \beta 1$ is believed to be involved in the interaction between mesenchymal stem cells and Col scaffolds (11). The $\alpha 2 \beta 1$ integrin inhibitor TCI-15 did not modify cell entrapment within the scaffolds. Although our findings fail to confirm the involvement of $\alpha 2 \beta 1$ integrin in binding of collagen scaffolds, they nevertheless suggest that other receptors for extracellular collagen (integrins $\alpha 1 \beta 1, \alpha 10 \beta 1 \alpha 11 \beta 1$ and Discoidin Domain Receptors) should be considered as potential targets for the interaction with extracellular collagen.

Our findings confirm that the tested samples of Col, either alone, with CS cross-linked by EDC or modified by DAC, are well tolerated by rat tissue and may be implanted into the brain. During the duration of the experiment, the Col fibers remained intact and hence may support cell migration and development. None of the tested biomaterials induced large glial scar formation. Moreover, no additional effects were seen when scaffolds seeded with embryonic nerve cells were implanted compared to the acellular grafts. This may be due to the possible death of all embryonic nerve cells after implantation of the scaffold. As our results show, the tested biomaterials are worth considering in the treatment of brain injury; however, additional modifications of the material are postulated to improve survival of the cells in vivo. This paper also confirmed the expression of integrins $\alpha 2$ and $\beta 1$ on nerve embryonic cells.

\section{Acknowledgements}

The authors would like to thank Mrs Teresa Staszewska (Department of Behavioral Pathophysiology, Medical University of Lodz, ul. Zeligowskiego 9/7, 90-752 Lodz, Poland) for her technical assistance. 


\section{Funding}

The present study was supported by National Science Centre (grant no. DEC-2011/03/B/ST8/05867). Publication of the present study was supported by the Medical University of Lodz (grant no. 503/6-103-02/503-061-001-18).

\section{Availability of data and materials}

The datasets used and/or analyzed during the current study are available from the corresponding author on reasonable request.

\section{Authors' contributions}

JD and KP contributed to the research concept and design of the current study. JD, KJ, LP, BP, JS and MS collected and assembled the data. JD, KJ, MS and JS analyzed and interpreted the data. JD, KP and KJ wrote the manuscript. JD, KP, $\mathrm{KJ}$, LP, BP, MS and JS critically revised the article. JD, KP, KJ, LP, BP, JS and MS approved the article.

\section{Ethical approval and consent to participate}

The experiments were approved by the Local Commission of Ethics in Lodz.

\section{Patient consent for publication}

Not applicable.

\section{Competing interests}

The authors declare that they have no competing interests.

\section{References}

1. Chwalek K, Tang-Schomer MD, Omenetto FG and Kaplan DL: In vitro bioengineered model of cortical brain tissue. Nat Protoc 10: 1362-1373, 2015

2. Huang KF, Hsu WC, Chiu WT and Wang JY: Functional improvement and neurogenesis after collagen-GAG matrix implantation into surgical brain trauma. Biomaterials 33: 2067-2075, 2012.

3. Teng YD, Lavik EB, Qu X, Park KI, Ourednik J, Zurakowski D, Langer R and Snyder EY: Functional recovery following traumatic spinal cord injury mediated by unique polymer scaffold seeded with neural stem cells. Proc Natl Acad Sci USA 99: 3024-3029, 2002.

4. Kowalska-Ludwicka K, Cala J, Grobelski B, Sygut D, Jesionek-Kupnicka D, Kolodziejczyk M, Bielecki S and Pasieka Z: Modified bacterial cellulose tubes for regeneration of damaged peripheral nerves. Arch Med Sci 9: 527-534, 2013.

5. Huang KF, Hsu WC, Hsiao JK, Chen GS and Wang JY: Collagen-Glycosaminoglycan Matrix implantation promotes angiogenesis following surgical brain trauma. BioMed Res Int 2014: 672409, 2014.

6. Bandtlow CE and Zimmerman DR: Proteoglycans in the developing brain: New conceptual insights for old proteins. Physiol Rev 80: 1267-1290, 2000.

7. Drobnik J, Pietrucha K, Piera L, Szymański J and Szczepanowska A: Collagenous scaffolds supplemented with hyaluronic acid and chondroitin sulfate used for wound fibroblast and embryonic nerve cell culture. Adv Clin Exp Med 26 223-230, 2017.

8. Pietrucha K, Szymański J and Drobnik J: The behavior of embryonic neural cells within the 3D micro-structured collagen-based scaffolds. IFMBE Proceedings 45: 549-552, 2015.
9. Sirko S, von Holst A, Wizenmann A, Gotz M and Faissner A: Chondroitin sulphate glycosaminoglycans control proliferation, radial glia cell differentiation and neurogenesis in neural stem/progenitor cells. Development 134: 2727-2738, 2007.

10. Li YY, Choy TH, Ho FC and Chan PB: Scaffold composition affects cytoskeleton organization cell-matrix interaction and the cellular fate of human mesenchymal stem cells upon chondrogenic differentiation. Biomaterials 52: 208-220, 2015.

11. Milner R and Campbel IL: The integrin family of cell adhesion molecules has multiple functions within CNS. J Neurosci Res 69: 286-291, 2002

12. Pietrucha K, Zychowicz M, Podobinska M and Buzanska L: Functional properties of different collagen scaffolds to create a biomimetic niche for neurally committed human induced pluripotent stem cells (iPSC). Folia Neuropathol 55: 110-123, 2017.

13. Pietrucha K: Physicochemical properties of 3D collagen-CS scaffolds for potential use in neural tissue engineering. Int J Biol Macromol 80: 732-739, 2015.

14. Pietrucha K: Development of collagen cross-linked with dialdehyde cellulose as a potential 3D scaffold for neural tissue engineering. IFMBE Proceeding 45: 349-352, 2015.

15. Pietrucha K and Safandowska M: Dialdehyde cellulose-crosslinked collagen and its physicochemical properties. Process Biochem 50: 2105-2111, 2015.

16. Pietrucha K, Marzec E and Kudzin M: Pore structure and dielectric behaviour of the 3D collagen-DAC scaffolds designed for nerve tissue repair. Int J Biol Macromol 92: 1298-1306, 2016.

17. Rauch U: Extracellular matrix components associated with remodeling process in brain. Cell Mol Life Sci 61: 2031-2045, 2004.

18. Jurga M, Dainiak MB, Sarnowska A, Jablonska A, Thripathi A, Plieva FM, Savina IN, Strojek L, Jungvid H, Kumar A, et al: The performance of laminin-containing cryogel scaffolds in neural tissue regeneration. Biomaterials 32: 3423-3434, 2011.

19. Vaysse L, Beduer A, Sol JC, Vieu C and Loubinoux I: Micropatterned bioimplant with guided neuronal cells to promote tissue reconstruction and improve functional recovery after primary motor cortex insult. Biomaterials 58: 46-53, 2015.

20. Ju R, Wen Y, Gou R, Wang Y and Xu Q: The experimental therapy on brain ischemia by improvement of local angiogenesis with tissue engineering in the mouse. Cell Transplant 23 (Suppl 1): S83-S95, 2014.

21. Yu S, Yao S, Wen Y, Wang Y, Wang H and Xu Q: Angiogenic microspheres promote neural regeneration and motor function recovery after spinal cord injury in rats. Sci Rep 6: 33428, 2016.

22. Tapon-Bretaudière J, Drouet B, Matou S, Mourão PA, Bros A, Letourner D and Fischer AM: Modulation of vascular human endothelial rat smooth muscle cell growth by a fucosylated chondroitin sulphate from echinoderm. Thromb Haemost 84: 332-337, 2000.

23. Deguchi K, Tsuru K, Hayashi T, Takaishi M, Nagahara M, Nagotani S, Sehara Y, Jin G, Zhang H, Hayakawa S, et al: Implantation of the new porous gelatin-siloxane hybrid into brain lesions as a potential scaffod for tissue regeneration. J Cereb Blood Flow Metab 26: 1263-1273, 2006.

24. Summitomo S, Muramatsu R, Fujii S and Yamashita T: Vascular endothelial cells promote cortical neurite outgrowth via an integrin $\beta 3$-dependent mechanism. Biochem Biophys Res Commun 450: 593-597, 2014.

25. Emgard M, Hallin U, Karlsson J, Bahr BA, Brundin P and Blomgren K: Both apoptosis and necrosis occur early after intracerebral grafting of ventral mesencephalic tissue: A role for protease activation. J Neurochem 86: 1223-1232, 2003.

26. Sortwell CE, Pitzer MR and Collier TJ: Time course of apoptotic cell death within mesencephalic cell suspension grafts: Implications for improving grafted dopamine neuron survival. Exp Neurol 165: 268-277, 2000.

27. Marchionini DM, Collier TJ, Pitzer MR and Sortwell CE: Reassessment of caspase inhibition to augment grafted dopamine neuron survival. Cell Transplant 13: 273-282, 2004.

28. Lanfer B, Hermann A, Kirsh M, Freudenberg U, Reuner U, Werner C and Storch A: Directed growth of adult human white matter stem cell-derived neurons on aligned fibrillar collagen. Tissue Eng Part A 16: 1103-1113, 2010.

29. Yoshinaga T, Hashimoto E, Ukai W, Ishii T, Shirasaka T, Kigawa Y, Tateno M, Kaneta H, Watanabe K, Igarashi T, et al: Effects of atelocollagen on neural stem cell function and its migrating capacity into brain in psychiatric disease model. J Neural Transm (Vienna) 120: 1491-1498, 2013.

This work is licensed under a Creative Commons Attribution-NonCommercial-NoDerivatives 4.0 International (CC BY-NC-ND 4.0) License. 